\title{
The Role of Accessory Obturator Arteries in Prostatic Arterial Embolization
}

\author{
Tiago Bilhim, PhD, MD, Joao Pisco, MD, PhD, \\ Luís Campos Pinheiro, MD, PhD, Hugo Rio Tinto, MD, \\ Lúcia Fernandes, MD, and José A. Pereira, MD
}

\begin{abstract}
In 9 of 491 patients $(1.8 \%$ ) who underwent prostatic arterial embolization (PAE) for benign prostatic hyperplasia from March 2009-November 2013, prostatic arteries arose from the external iliac artery via an accessory obturator artery (AOA). Computed tomography angiography performed before the procedure identified the variant and allowed planning before the procedure. The nine AOAs were catheterized from a contralateral femoral approach. Bilateral PAE was technically successful in the nine patients. There was a mean decrease in international prostate symptom score of 6.5 points and a mean prostate volume reduction of $15.1 \%$ (mean follow-up, $4.8 \mathrm{mo}$ ) in the nine patients.
\end{abstract}

\section{ABBREVIATIONS}

$\mathrm{AOA}=$ accessory obturator artery, DSA = digital subtraction angiography, IPSS = International Prostate Symptom Score, $\mathrm{PAE}=$ prostatic arterial embolization, $\mathrm{PVA}=$ polyvinyl alcohol, $\mathrm{QoL}=$ quality of life

Prostatic arterial embolization (PAE) to relieve lower urinary tract symptoms in patients with benign prostatic hyperplasia is a challenging procedure partly because of the highly variable origin and complex anatomy of the prostatic arteries (1). Computed tomography (CT) angiography performed before PAE has been used to help identify the prostatic arteries and to guide the procedure (2). The prostatic arteries may arise from the different branches of the internal iliac artery, including from the obturator artery, in $13 \%$ of pelvic sides $(1,2)$. However, the obturator artery may arise from the inferior epigastric artery or directly from the external iliac artery and be named an accessory or

From the Departamentos Universitários de Anatomia (T.B.), Radiologia (T.B., H.R.T., L.F.), and Urologia (L.C.P.), Faculdade de Ciências Médicas, Universidade Nova de Lisboa, Campo Mártires da Pátria 130, Lisbon, 1169-056; Interventional Radiology (T.B., J.P., H.R.T., L.F., J.A.P.), Hospital Saint Louis, Lisbon; and Urology Department (L.C.P.), Hospital São José, Centro Hospitalar de Lisboa Central, Lisbon, Portugal Received December 27, 2013; final revision received and accepted March 4, 2014. Address correspondence to T.B.; E-mail: tiagobilhim@hotmail.com

T.B. and J.P. are paid consultants for Cook Medical. H.R.T. is a paid consultant for Cook Medical and Celonova. None of the other authors have identified a conflict of interest.

Video 1 is available online at www.jvir.org.

(c) SIR, 2014

J Vasc Interv Radiol 2014; 25:875-879

http://dx.doi.org/10.1016/j.jvir.2014.03.005 aberrant obturator artery (AOA). An AOA is a common variation that may be present in $30 \%$ of pelvic sides (3). Prostatic arteries arising from AOAs are a possible variant of particular relevance when performing PAE. The purpose of this report is to clarify this anatomic variation and report successful catheterization and embolization in nine patients with lower urinary tract symptoms related to benign prostatic hyperplasia.

\section{MATERIALS AND METHODS}

The institutional review board waived the need to obtain permission to perform this retrospective analysis. From March 2009-November 2013, PAE was performed in 491 patients to relieve lower urinary tract symptoms secondary to benign prostatic hyperplasia. Nine patients with prostatic arteries arising from AOAs were included. AOAs were classified as (i) obturators arising from the inferior epigastric artery, (ii) obturators arising directly from the external iliac artery, or (iii) codominant obturators originating from both the external and the internal iliac artery systems (3).

Among the nine patients evaluated, mean patient age was 62.9 years (range, 51-74 y). Mean International Prostate Symptom Score (IPSS) was 18.9 points (range, 14-21 points), mean quality of life (QoL) score was 4.0 points (range, $3-6$ points). Mean peak flow rate was $11.2 \mathrm{~mL} / \mathrm{s}$ (range, $5.9-13.5 \mathrm{~mL} / \mathrm{s}$ ), mean postvoid residual 
urine was $108.0 \mathrm{~mL}$ (range, $22-274 \mathrm{~mL}$ ), mean prostatespecific antigen level was $4.2 \mathrm{ng} / \mathrm{mL}$ (range, $0.6-10.7 \mathrm{ng} /$ $\mathrm{mL}$ ), and mean prostate volume was $78.9 \mathrm{~cm}^{3}$ (range, 52-155 $\mathrm{cm}^{3}$ ). None of the patients were experiencing acute urinary retention before PAE.

All patients underwent CT angiography before PAE as previously described (1). PAE was performed under local anesthesia by a contralateral femoral approach; when the prostatic artery originated from the right AOA, the left femoral artery was punctured and vice versa. A Roberts uterine catheter (Cook, Inc, Bloomington, Indiana) was used in every patient to catheterize the inferior epigastric artery.

Digital subtraction angiography (DSA) of the inferior epigastric artery was performed in a contralateral anterior oblique projection ( 25 degrees). The AOA and prostatic artery were identified with CT angiography and DSA and catheterized with 2.4-F to 2.7-F microcatheters (Cantata; Cook, Inc; Progreat; Terumo, Tokyo, Japan) and 0.016-inch guide wires (Sagitta-16 Wire Guide; Cook, Inc; Glidewire GT; Terumo). Selective DSA of the prostatic artery $(4-5 \mathrm{~mL}$; at $2 \mathrm{~mL} / \mathrm{s})$ in neutral and contralateral anterior oblique (25 degrees) projections was performed before and after embolization.

Bilateral PAE was performed with $100-\mu \mathrm{m}$ polyvinyl alcohol (PVA) particles (Cook, Inc) $(\mathrm{n}=1$ patient), 100$\mu \mathrm{m}$ and $200-\mu \mathrm{m}$ PVA particles $(\mathrm{n}=4$ patients), $200-\mu \mathrm{m}$ PVA particles ( $\mathrm{n}=1$ patient), 300- to $500-\mu \mathrm{m}$ tris-acryl microspheres (Embosphere; BioSphere Medical, Rockland, Massachusetts) ( $\mathrm{n}=1$ patient), $300-$ to $500-\mu \mathrm{m}$ spherical PVA particles (Bead Block; Terumo) $(\mathrm{n}=1$ patient), and $400-\mu \mathrm{m}$ Polyzene-coated microspheres (Embozene; CeloNova BioSciences, Inc, Peachtree City, Georgia) ( $\mathrm{n}=1$ patient). The endpoint of embolization was occlusion of the identifiable vessels supplying the prostate and reflux toward the prostatic artery origin or into the epigastric artery.

Patients were evaluated before and 1, 3, 6, and 12 months after PAE with IPSS and QoL questionnaires, fluxometry, prostate-specific antigen, and prostate volume with transrectal ultrasound (4).

\section{RESULTS}

During the study time period, 9 of 491 patients $(1.8 \%)$ had prostatic arteries arising from an AOA. There was no case of prostatic arteries arising from an AOA on both pelvic sides. In seven patients, the prostatic artery arose from an AOA on the left pelvic side, and in 2 patients, the prostatic artery arose from an AOA on the right pelvic side. In seven patients, the AOA arose from the inferior epigastric artery $(78 \%)$, and in 2 patients, the AOA arose directly from the external iliac artery $(22 \%)$. There were no patients with codominant AOAs from both internal and external iliac artery systems. The prostatic artery origin from the AOA was identified with CT angiography before PAE and confirmed during the procedure in all 9 patients (Fig 1a-d).

The prostatic artery arising from the AOA was the central gland or anterolateral prostatic artery in all patients (Fig 2a-d, Video 1 [available online at $w w w$. jvir.org]) (1). In four patients, this was the single prostatic artery from that pelvic side, whereas in the remaining five patients, a posterolateral or caudal prostatic artery was identified arising from the internal iliac artery system (internal pudendal artery in three patients; inferior gluteal artery in two patients) (1).

Mean procedural time was 83.3 minutes (range, 50 $120 \mathrm{~min}$ ), and mean fluoroscopy time was 28.0 minutes (range, 12.4-59.8 min). All patients were able to void spontaneously after PAE. No severe pain was reported during or after PAE. One patient reported slight selflimited hematuria in the first 3 days after PAE. There were no other major or minor adverse events according to the Society of Interventional Radiology (SIR) criteria (5).

Mean follow-up time was 4.8 months (range, 1-12 mo): 1 month in one patient; 3 months in four patients; 6 months in three patients, and 12 months in one patient. There was a mean decrease in IPSS of 6.5 points and in QoL severity score of 2 points. After PAE, the mean IPSS/QoL severity score was $12.4 / 2$ points. Mean prostate volume reduction was $11.9 \mathrm{~cm}^{3}(15.1 \%)$, mean prostate-specific antigen reduction was $1.0 \mathrm{ng} / \mathrm{mL}$ $(23.8 \%)$, mean peak flow rate increase was $2.8 \mathrm{~mL} / \mathrm{s}$ $(25 \%)$, and mean postvoid residual urine reduction was $84.7 \mathrm{~mL}(78.4 \%)$.

\section{DISCUSSION}

This work highlights the need to look for an AOA when performing PAE, especially when no prostatic arteries are identified inside the pelvis arising from the internal iliac artery branches. AOAs are familiar to most interventional radiologists, and they should be looked for after pelvic trauma because they may be the source of bleeding (6). AOAs have also been given the name of "corona mortis"; however, there is no consensus on the definition of the corona mortis. Some authors consider the corona mortis to be limited to AOAs, whereas others consider it to be any venous or arterial communication between the external and internal iliac systems through the obturator vessels $(3,6)$. In this study, AOAs were defined as any obturator artery arising from the inferior epigastric artery $(78 \%)$ or directly from the external iliac artery $(22 \%)(3,6)$. None of the reported patients had codominant or anastomotic obturator arteries from both internal and external iliac artery systems.

Cadaveric and imaging studies have described in detail the prostatic artery anatomy relevant for embolization 

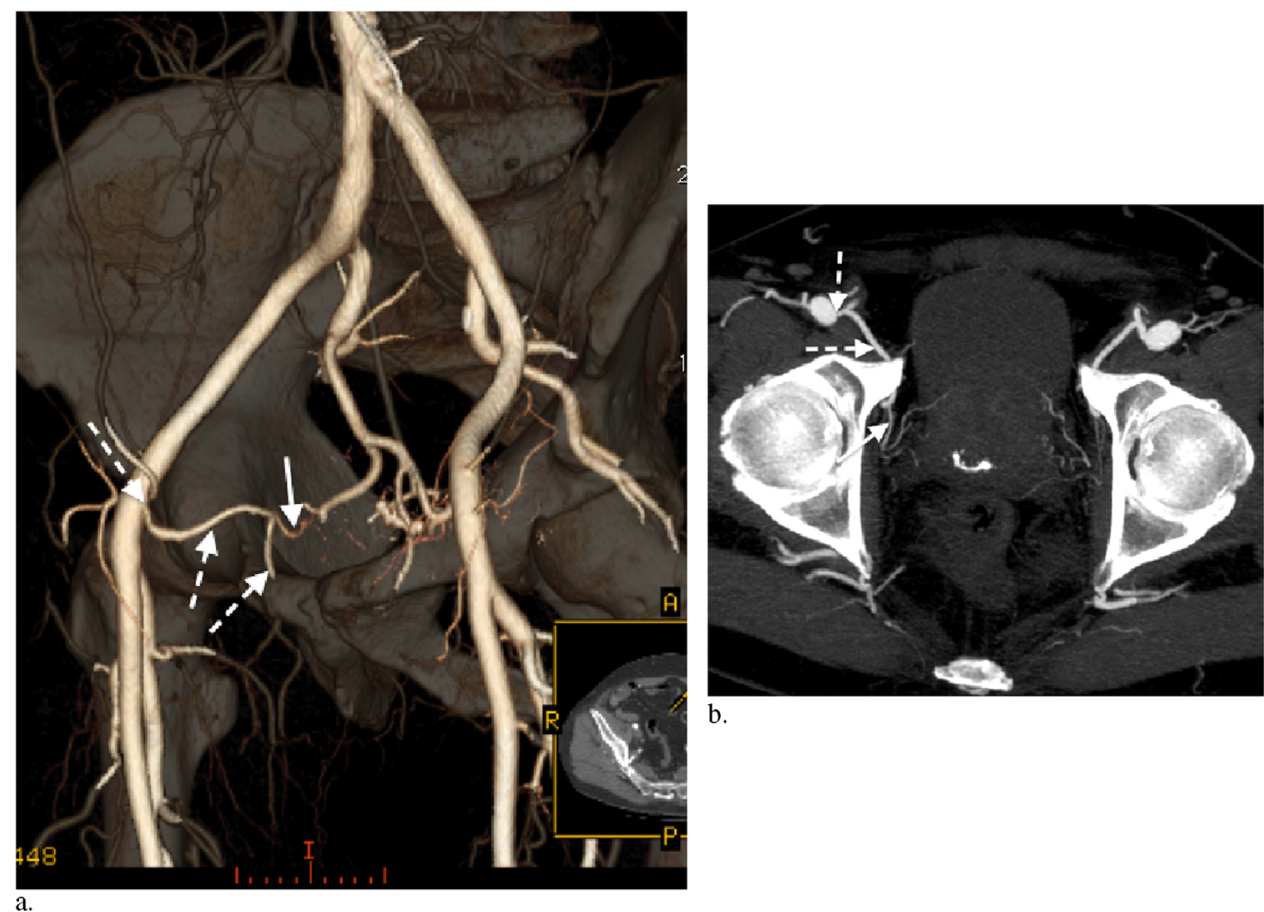

b.
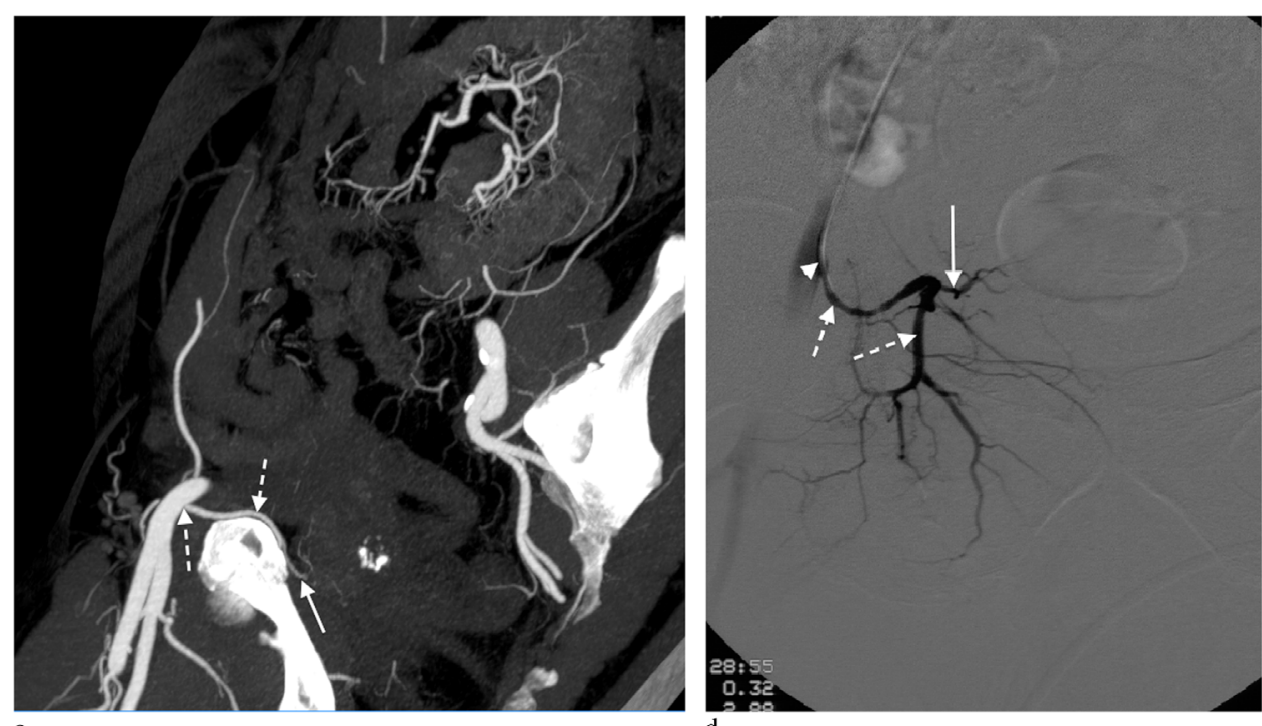

d.

Figure 1. Single prostatic artery (solid arrow) arising from an AOA (dashed arrows) directly from the external iliac artery on the right pelvic side. (a) CT angiography volume rendering reformat. (b) CT angiography axial minimum intensity projection reformat. (c) CT angiography coronal curved minimum intensity projection reformat. (d) Selective DSA of the right AOA in neutral position. Note the Roberts uterine catheter (arrowhead) through a contralateral femoral approach.

$(1,2,7,8)$. Prostatic arteries have highly variable origins from the branches of the internal iliac artery. The rare incidence of prostatic arteries arising from AOAs $(<2 \%$ from our experience) may explain why it was not reported before. Previous studies on prostatic artery anatomy included $<75$ patients $(1,2,7,8)$, which was probably the reason for not reporting this variant.

This study also reinforces the role of imaging planning for PAE before the procedure. The presence of prostatic arteries arising from AOAs outside the pelvis could lead to an increase in procedural and fluoroscopy times or to unilateral embolization procedures. However, the mean procedural and fluoroscopy times were comparable to previously reported results (4), and bilateral PAE was achieved in every patient; we believe this to be due to the use of CT angiography before PAE.

The contralateral femoral artery was punctured to facilitate catheterization of the AOA. Ipsilateral femoral puncture could have led to failed catheterization of the AOA. Through contralateral femoral access, all inferior epigastric arteries were catheterized with a Roberts uterine catheter. Contrary to the standard ipsilateral oblique view used in the pelvis during internal iliac artery DSA before PAE (1), the contralateral anterior 

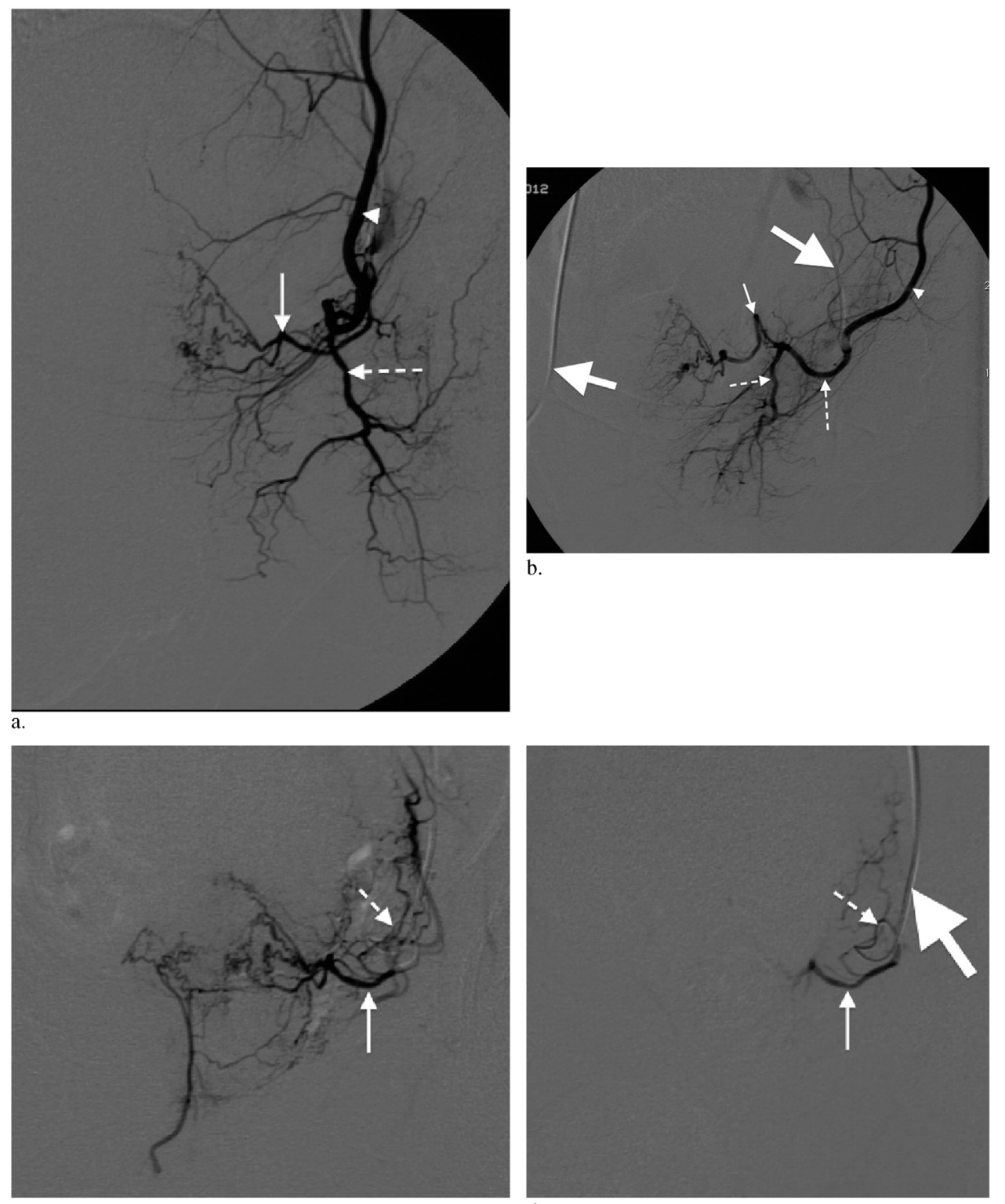

c.

d.

Figure 2. Prostatic artery (solid arrows) arising from an AOA (dashed arrows) from the inferior epigastric artery (arrowhead) on the left pelvic side. (a) Selective DSA of the left inferior epigastric artery in neutral position. (b) Selective DSA of the left inferior epigastric artery in contralateral anterior oblique (25 degrees) projection. Note the Roberts uterine catheter (large arrows) through a contralateral femoral approach. The three arteries are easier to depict in this projection. (c) Selective prostatic artery DSA in neutral projection after selective catheterization with a microcatheter (dashed arrow). (d) Angiogram obtained after embolization.

oblique view ( 25 degrees) proved to be more useful to depict the inferior epigastric artery, AOA, and prostatic artery correctly. In this projection, the AOA and prostatic artery are well depicted, and selective prostatic artery catheterization becomes easier with the use of a microcatheter.

Although the number of patients is small, the clinical results reported are similar to larger series (4). As long as the prostatic artery is correctly identified and embolization is performed correctly, no significant complications are expected - from untargeted embolization to the abdominal wall, lower limbs, bladder, rectum, or penis.

This study has some limitations. We evaluated only patients with prostatic arteries arising from AOAs and not all patients with AOAs. Our goal was to highlight the possibility of prostatic arteries arising from outside the pelvis and not to describe AOAs, as previously reported (3). The retrospective nature of the analysis, in a small cohort of patients with a short and variable follow-up time, is an additional limitation to the interpretation of these results. During this study (4-year duration), we were able to identify only nine patients with prostatic arteries from AOAs $(<2 \%)$. The use of different embolic agents reflects the long time period of this study.

In conclusion, when performing PAE, interventionalists should be aware that $<2 \%$ of patients have prostatic arteries arising from AOAs outside the pelvis. Imaging 
planning with CT angiography before a procedure correctly identifies this variant and may help guide PAE.

\section{REFERENCES}

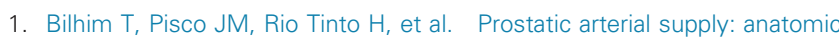
and imaging findings relevant for selective arterial embolization. J Vasc Interv Radiol 2012; 23:1403-1415.

2. Bilhim T, Pisco JM, Furtado A, et al. Prostatic arterial supply: demonstration by multirow detector angio CT and catheter angiography. Eur Radiol 2011; 21:1119-1126.

3. Smith JC, Gregorius JC, Breazeale BH, Watkins GE. The corona mortis, a frequent vascular variant susceptible to blunt pelvic trauma: identification at routine multidetector CT. J Vasc Interv Radiol 2009; 20:455-460.
4. Pisco J, Campos Pinheiro L, Bilhim T, et al. Prostatic arterial embolization for benign prostatic hyperplasia. Short and medium term results. Radiology 2013; 266:668-677.

5. Sacks D, McClenny TE, Cardella JF, Lewis CA. Society of Interventional Radiology clinical practice guidelines. J Vasc Interv Radiol 2003; 14: S199-S202.

6. Johnson GE, Sandstrom CK, Kogut MJ, et al. Frequency of external iliac artery branch injury in blunt trauma: improved detection with selective external iliac angiography. J Vasc Interv Radiol 2013; 24:363-369.

7. Bagla S, Rholl KS, Sterling KM, et al. Utility of cone-beam CT imaging in prostatic artery embolization. J Vasc Interv Radiol 2013; 24 1603-1607.

8. Garcia-Monaco R, Garategui L, Kizilevsky N, Peralta O, Rodriguez P, Palacios-Jaraquemada J. Human cadaveric specimen study of the prostatic arterial anatomy: implications for arterial embolization. J Vasc Interv Radiol 2014; 25:315-322. 